\title{
Hump-Shaped Broadband Noise on a Fan at Off-Design Conditions
}

\author{
Manuel Henner, François Franquelin, Bruno Demory, Youssef Beddadi, Charles Roland, Aurélien Serran \\ Valeo Thermal Systems 8, rue Louis Lormand ZA de l'Agiot, La Verrière 78321, Frances.
}

Received: April 22, 2015 / Accepted: May 20, 2015 / Published: September 25, 2015.

\begin{abstract}
A spurious effect of blowing noise is sometimes detected on fans working at high flow rate, showing a large hump around $4000 \mathrm{~Hz}$ on the acoustic spectra. A Tollmien-Shlichtling (TS) effect is suspected, and several compressible LES are made on aerodynamic profiles to detect and investigate this phenomenon. Vortex shedding is observed at the trailing edge, triggering some density fluctuations when a thin boundary layer exists on the profile upper side. Acoustic waves with length equivalent to the chord are observed. A profile modification is proposed with a camber brought closer to the leading edge. This shape tested on a prototyped fan confirms the weakening of the TS effect.
\end{abstract}

Key words: Hump-shaped Broadband noise, Axial Fan , LES compressible simulation, Tollmien-Shlichtling

\section{Introduction}

Axial fan systems are used in cars to force the flow through the stacking of heat exchangers placed at the vehicle front end. They are designed to provide a low pressure rise with high flow rates, especially when the vehicle is moving, and their power is limited by the electrical power available on the 12 volt circuit. As a consequence of the low torque requirement, the fan is characterized by a low solidity, and each blade operates almost like an isolated airfoil. This characteristic presents some advantages for acoustics as blade to blade interactions does not exist. Fan self-noise can be therefore estimated with models based on the Amiet's theory, and such investigations related to fan applications have been widely reported, for instance in [1] and [2]. Both broadband and tonal noises are in the automotive industry subject to intense studies by car makers and Original Equipment Makers (OEM). Both of them should provide solutions respecting the environmental requirement for a low level of noise in

Corresponding author: Franquelin François, Valeo Thermal Systems 8, La Verrière 78321, Frances. E-mail: francois.franquelin.ext@valeo.com. urban conditions.

The validation process for fan noise shows occasionally a spurious phenomenon which creates an additional blowing noise on the fan, not predicted by traditional acoustic models. This disturbance is easily visible by post-processing the recorded sound in the frequency domain. The sound pressure level shown in Figure 1 presents such a result. Spectrum has been obtained in an half anechoic chamber with a microphone placed on the rotation axis at $1 \mathrm{~m}$ in front of the fan. If the tonal peaks on the broadband noise are classical results for automotive fan systems, the broadband noise with a hump around $4000 \mathrm{~Hz}$ is however seldom seen. When this phenomenon occurs, the concerned fan is rejected as it creates an unpleasant blowing noise, and it leads to a $5 \mathrm{dBA}$ increased sound level.

Further investigations were conducted, and it was observed that this effect occurs when the fan operates at its highest flow rate (zero pressure condition), and disappears progressively when the flow rate decreases. To complete the observations, several experiments with rubber tapes positioned on the blade upper side have shown a minimization of the phenomenon, with 


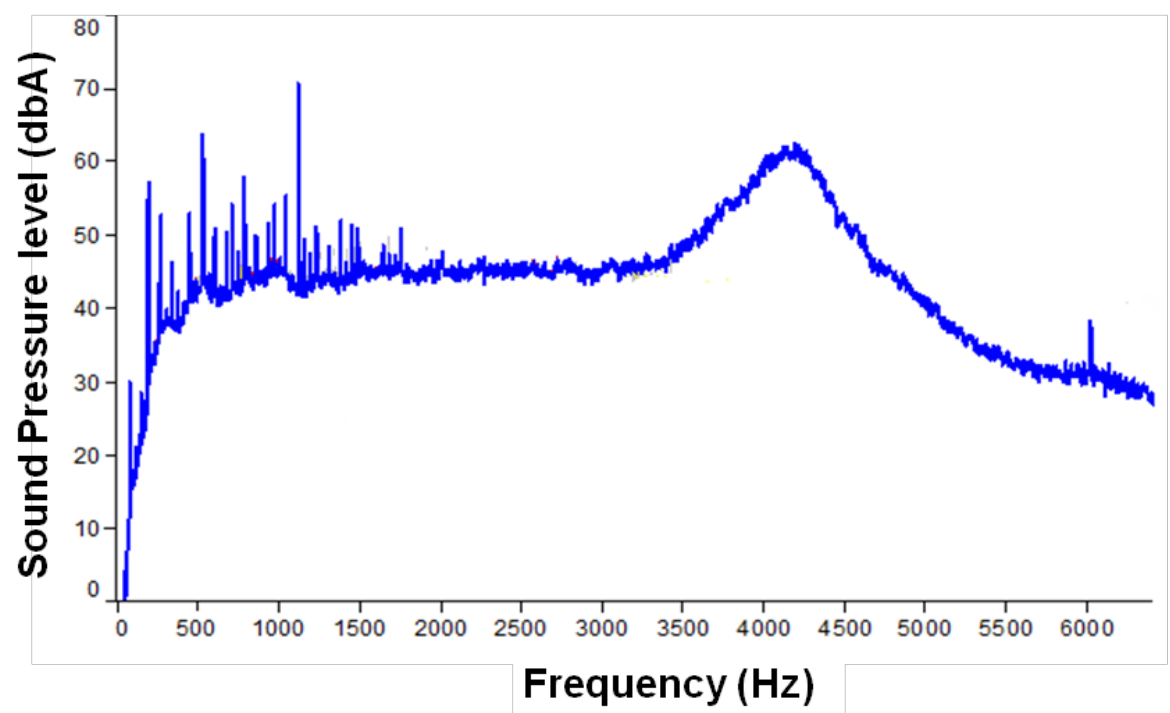

Fig. 1 Sound pressure level with hump shaped broadband noise.

a important shift towards lower frequencies, i.e around $3000 \mathrm{~Hz}$.

A Tollmien-Shlichtling (TS) effect is obviously suspected, and should be avoided in future fan designs. This paper presents investigations made by numerical simulation to isolate the phenomenon and to provide some data on its mechanism. A modification of the blade profile is also proposed and investigated.

\section{TS Phenomenon on Rotating Machines}

The phenomenon firstly described by Tollmien and Shlichtling involves an aeroacoustic interaction between a profile and its wake. An oscillation is observed at a fixed frequency at the trailing edge, and this latter retroacts on the boundary layer developed on the blade upper side. For given conditions with low flow incidence on the profile, transition to turbulence occurs at the same frequency and creates acoustic waves that further excite the oscillation in the wake. A self-maintenance mechanism occurs after its triggering. When the coupling is strong enough an acoustic peak is observed. Details on this mechanism can be found in [3]. Both conditions to be found are an excitation at trailing edge and a thin boundary layer with a transition from laminar to turbulent. This phenomenon is therefore dependent on the Reynolds number and the considered profile.

The TS phenomenon is traditionally described for isolated airfoils in wind-tunnel conditions, and these experiments are used to explain identical effects on practical applications, as for instance the noise produced by a Katana as reported by Roger in [4]. For automotive fan, it has already been observed and described by Longhouse [5], who developed a visualization technique with naphthalene deposition to follow the friction lines until the transition to turbulent flow. His experimentation is confirmed by the one presented in this paper with a different geometry, but for almost identical conditions (similar Reynolds and experimental conditions).

Operating point of the fan showing TS phenomenon is $172 \mathrm{~Pa}$ at $2797 \mathrm{~m} 3 / \mathrm{h}$ for a rotational speed of 2200 rpm with an efficiency of $43.5 \%$. Its aerolic diameter is $480 \mathrm{~mm}$ with forward sweep at the bottom and a backward sweep at the top (cf. Figure 2).

A recording of the sound during a velocity ramp-up has provided some prima facie evidence of a TS effect: in Figure 3, the average frequency of the hump of noise varies as a function of the power of 1,15 as expected theoretically. The classical staged evolution with local variations to the power of 0,8 (roughly assessed between 0.85 and 0.87 in our case) is 


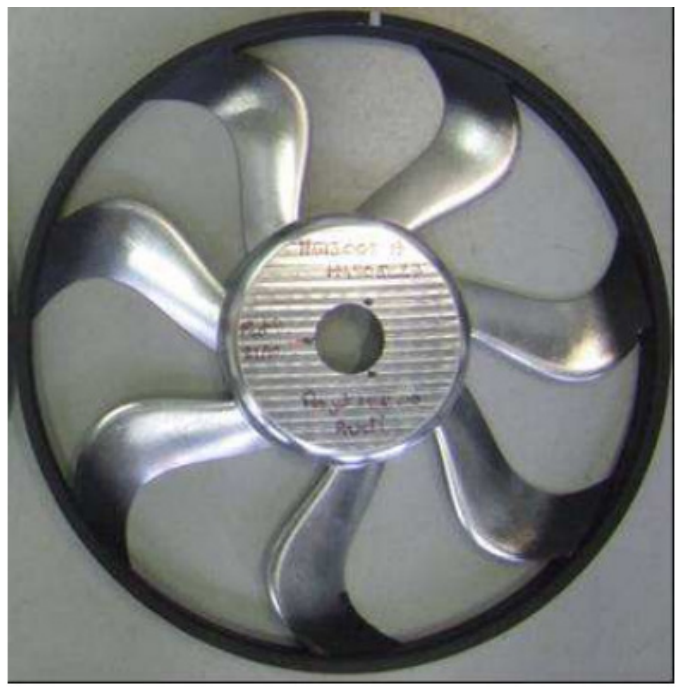

Fig. 2 Tested fan (forward-backward sweep).

however not obvious, probably hidden behind the quite large hump shaped acoustic phenomenon [6].

In the case of a turbomachine like the fan for automotive application, both blade profile and Reynolds number are changing with the radius, yielding to various conditions of TS along the span. As a consequence, a first assumption would be to explain the wide hump in the frequency domain by the merge of several peaks of frequency corresponding each to a local TS phenomenon. The numerical investigations presented in the next paragraph will provide some hints about the nature of this hump shaped broadband noise.

\section{Numerical Investigations}

Investigations are conducted in two steps, aimed at first to analyze the fan behavior, and secondly to simulate the acoustic wave. In both cases, simulations are done with the commercial code Star-CCM+ 7.04, in two different ways:

- The complete fan is modeled for a RANS simulation, with the experimental condition of high flow rate, close to the zero pressure point. The post-processing extracts the local conditions on the blade, i.e. the air velocity and the inlet flow angle at a given radius.

- A compressible LES simulation is made for a blade portion with these velocity conditions (magnitude and angle). The geometry is extracted at location where the radial velocity is close to zero in order to have realistic conditions. The blade profile is developed on a plane, and extruded by a tenth of its chord.

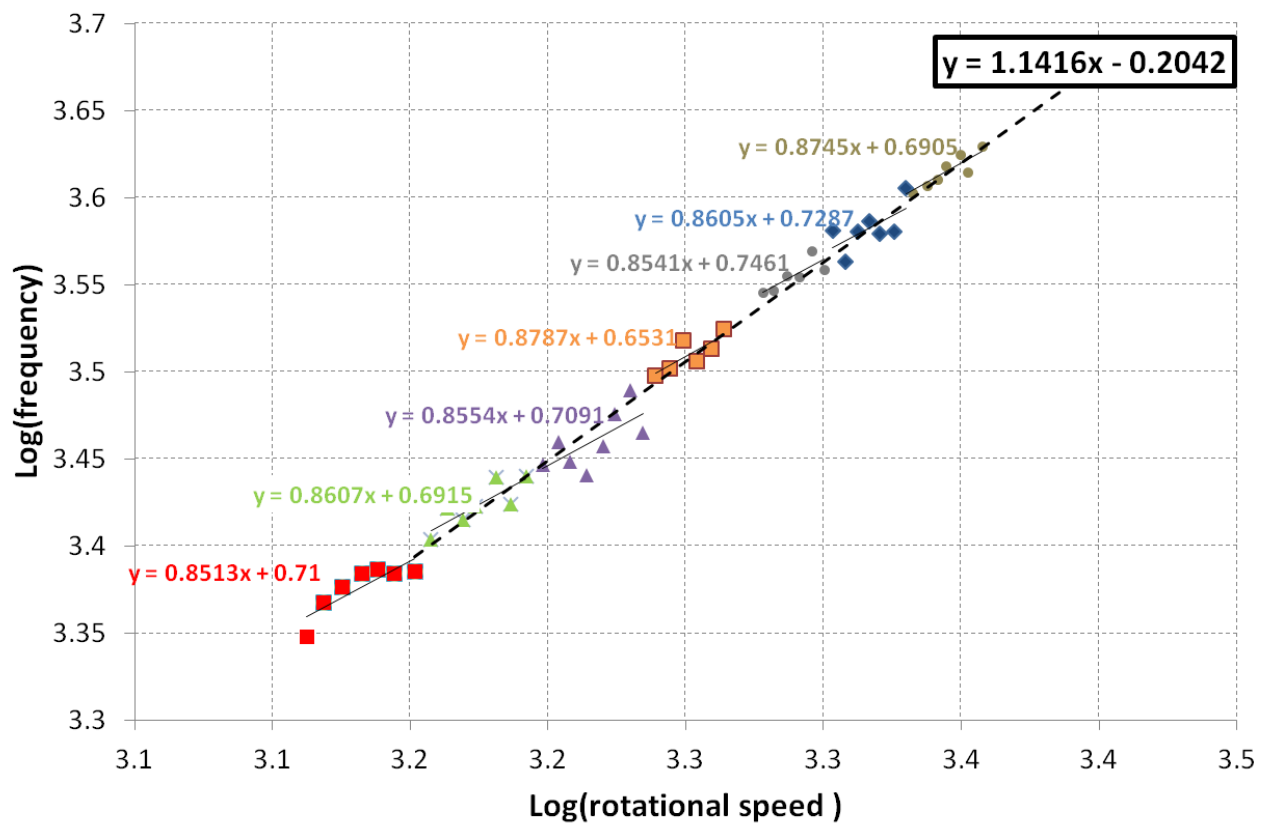

Fig. 3 Log of the hump shaped frequency in function of the log of the rotational speed. 


\subsection{D RANS Simulation}

The fan simulation is performed using a polyhedral mesh, with cell extrusion on the wall able to fulfill the requirement of a $\mathrm{Y}+$ close to 1 . The standard SST k- $\omega$ turbulence model and a multiple reference frame are used, according to our validated simulation methodology presented in [7]. As expected for the high flow rate simulated, the blade is working at a slight under-incidence condition, yielding to a thin boundary layer on the upper side and a separation on the lower side. Vorticities assessed by the turbulence model are presented in Figure 4 to present the subsequent boundary layers of both profile sides. In combination with the thick trailing edge (thickness is a constraint imposed by the process for the plastic injection), it creates a large wake behind the profile. Due to the steady nature of the simulation, no coherent structure could be observed at this stage. However, same observations about the structure of the flow can be made all along the span despite the velocity change with the blade radius.

Regarding the radial velocity distribution presented in Figure 5, the profile located a $2 / 3$ of the span is selected because the radial velocity component is minimal and close to zero.

\subsection{LES Compressible Simulation}

A limited domain is used to do a compressible LES in a cascade condition. Figure 6 presents the domain where the profile, the inlet, the outlet and two periodic conditions can be seen. The first one cuts two consecutive blade to blade passages, creating thus a periodic channel around the profile. The second one is used to limit the domain in the spanwise direction, while allowing the development of the turbulence with periodic structures.

The Figure 7 presents the mesh created, using about 30 million polyhedral cells and 19 cell extrusions from the wall. The Wall-Adapting Local Eddy-viscosity (WALE) model is used for the sub-grid modeling, and the simulation is made for physical time of 6.10-2

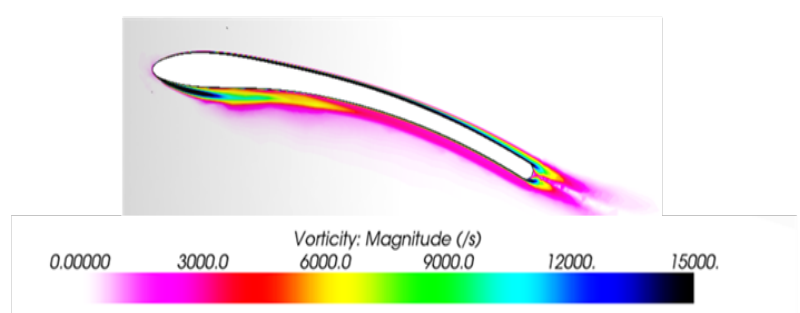

Fig. 4 Vorticity around blade profile in full fan simulation (RANS).

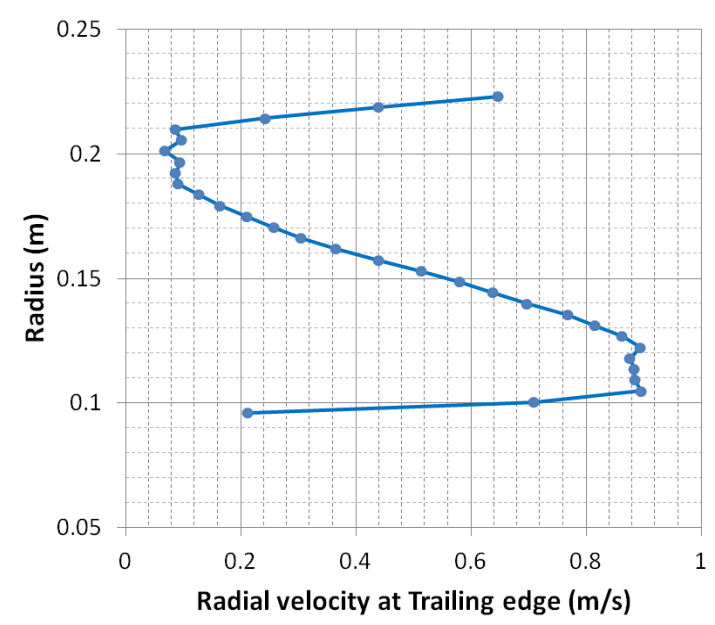

Fig. 5 Radial velocity at trailing edge along the span.

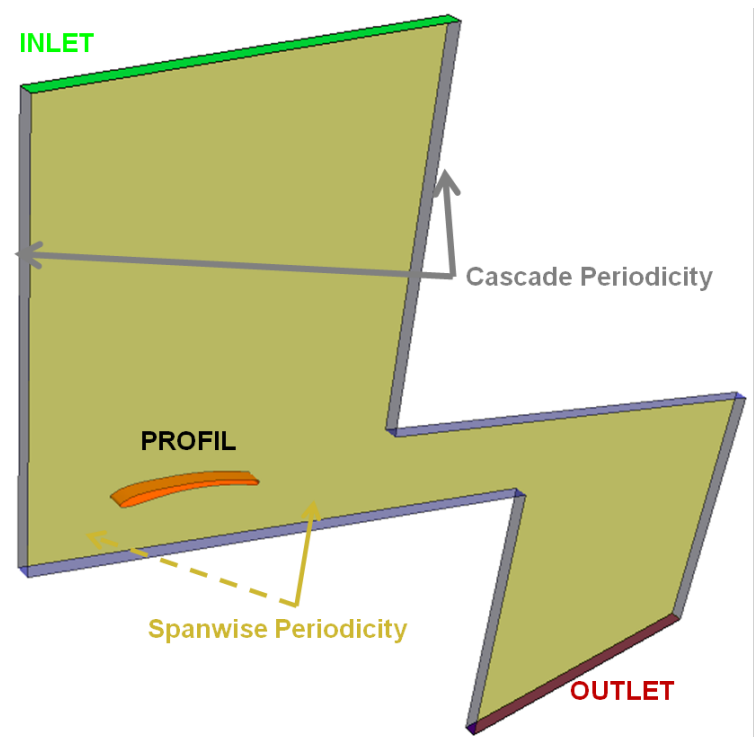

Fig. 6 Domain of simulation for the LES.

seconds. Boundary condition at inlet is defined with a velocity magnitude, an angle and with self turbulence based on WALE coefficient [8] (Von Karman constant: 0.41 ; Subgrid viscosity coefficient: 0.544 ; 

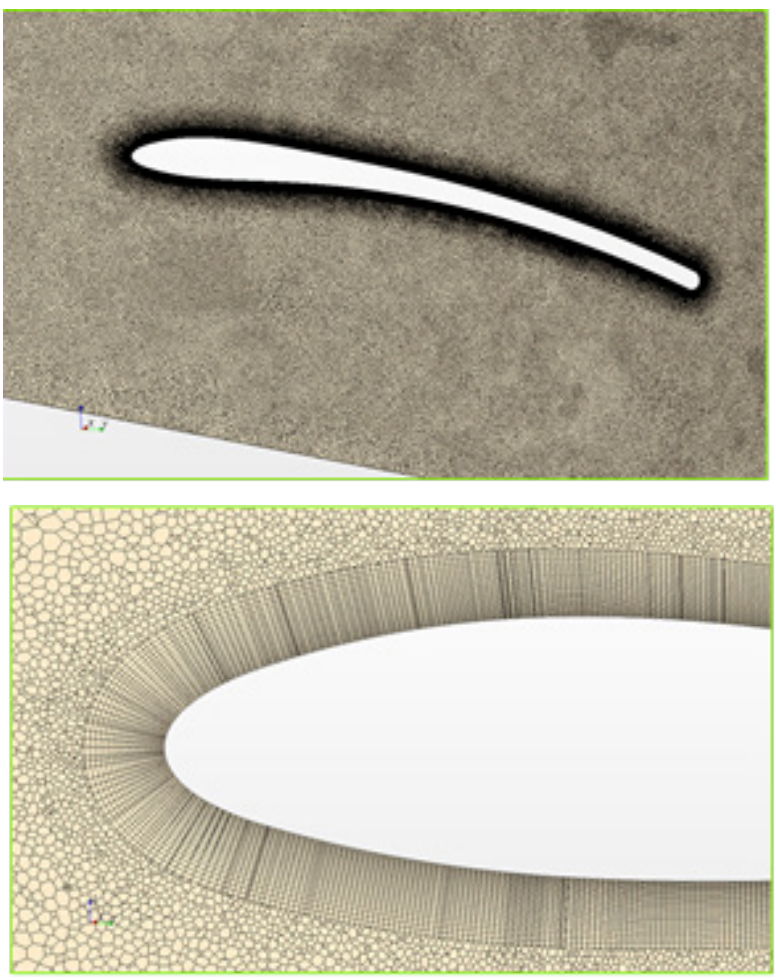

Fig. 7 Mesh used for LES.

Time scale coefficient: 1.25) .The small time step (3.10-6 seconds allows a theoretical frequency cut-off of $167 \mathrm{kHz}$. Global monitoring of relevant scalars (pressure, velocities) in the flow are used to ensure that numerical transient effects are gone before doing the final flow analysis. In the end, the last 6.10-3 second is used for post-processing.

Result can be seen in Figure 8 in terms of vorticity. It confirms that the profile is working with a slight under-incidence, and that a thick boundary layer develops on the lower side. However, the separation close to the leading edge is strongly reduced, whereas the upper side shows a very thin boundary layer. Thanks to the unsteady simulation, the development of coherent vortical structures can be observed at the trailing edge. They are further convected in the wakes without merging because of the large distance between two consecutive blades, as presented in Figure 9. It confirms that profiles can be considered as isolated airfoils for the acoustics, but not for the flow deviation which is bigger.

The frequency of the vortex shedding observed at

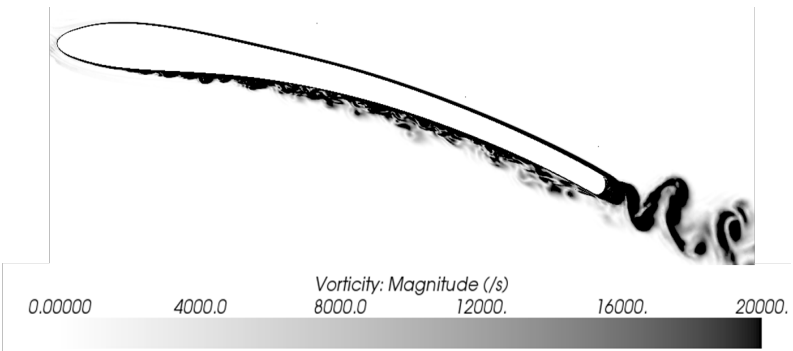

Fig. 8 Vorticity around blade profile in LES.

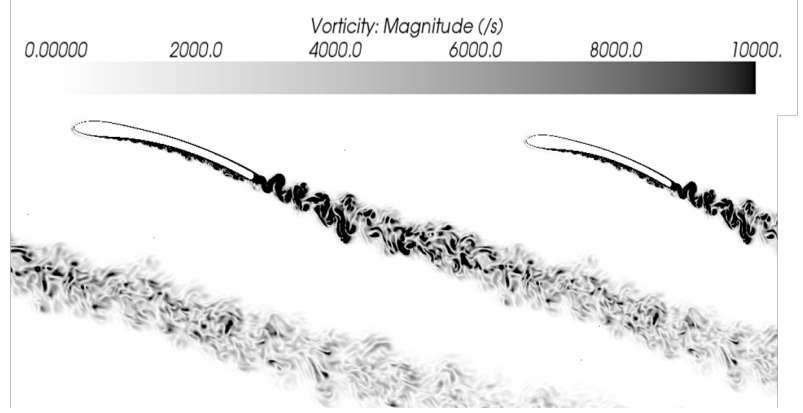

Fig. 9 Vorticity in the wakes.

trailing edge has been measured at $5000 \mathrm{~Hz}$, which is not exactly the average frequency of the hump measured experimentally around $4200 \mathrm{~Hz}$. This shift could be however explained either by the full 3D effect on the fan (various Reynolds number from blade to top), or by the assumption made by extracting RANS data and implementing them in a LES. Although this discrepancy, it must be noted that the Fast Fourier Transform of the pressure monitored at 1.5 chord behind the trailing edge (in the wake) shows also a wide hump in the frequency domain, visible in Figure 10. It has some similarities with the acoustic spectrum previously shown in Figure 1.

This acoustic phenomenon is also visible in a different way by using a visualization of the density fluctuation along time. In Figure 11, two snap-shoots of the density are presented, both being separated by half a period of a $5000 \mathrm{~Hz}$ frequency phenomenon. A fluctuation appears on two thirds of the upper side of the profile, and can be identified as a dipolar source emitting at the same frequency than the vortex shedding.

Further evidence of the acoustic effect is done with the velocity divergence which shows the compressibility effect and the acoustic waves. In Figure 12, two main 


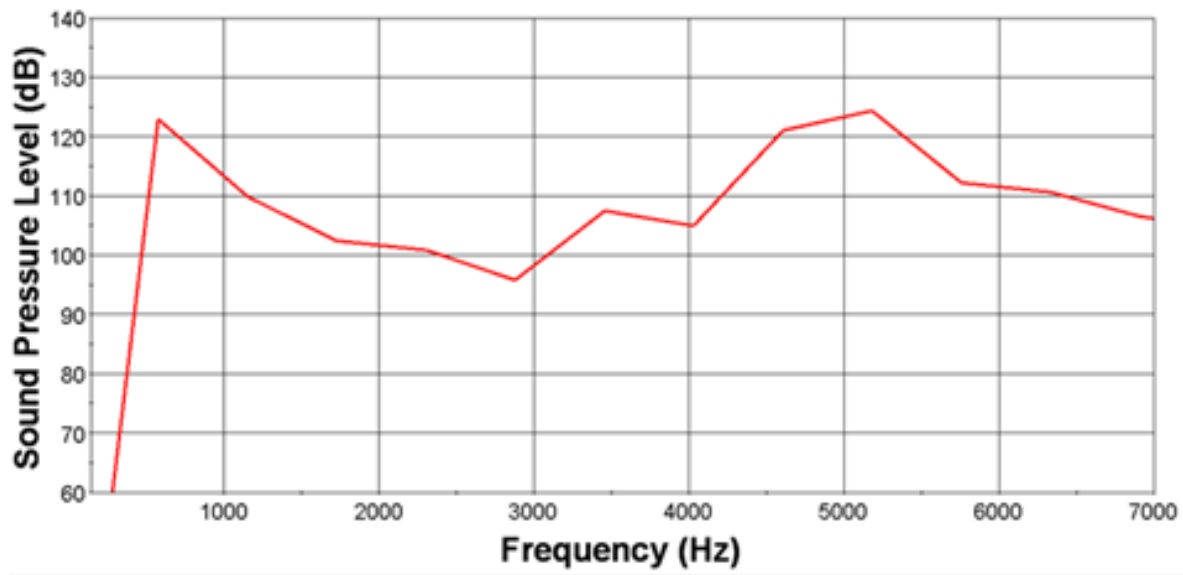

Fig. 10 Sound pressure level in the wake 1.5 chord downstream.
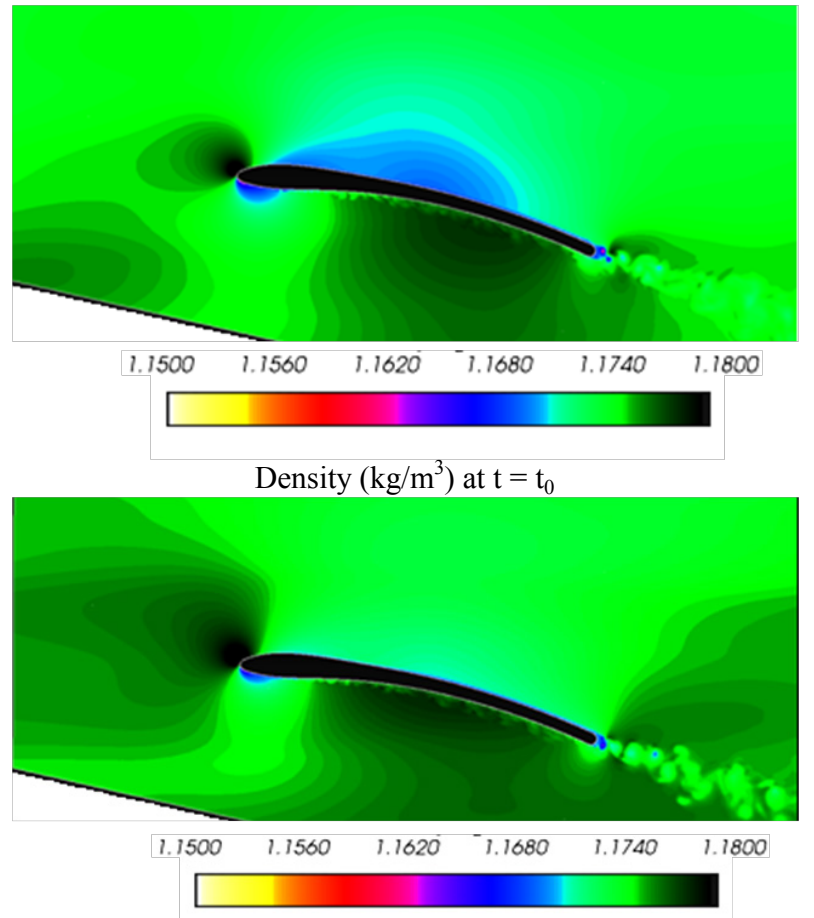

Density $\left(\mathrm{kg} / \mathrm{m}^{3}\right)$ at $\mathrm{t}=\mathrm{t}_{0}+2.10^{-4} \mathrm{~s}$

Fig. 11 Density periodic fluctuation.

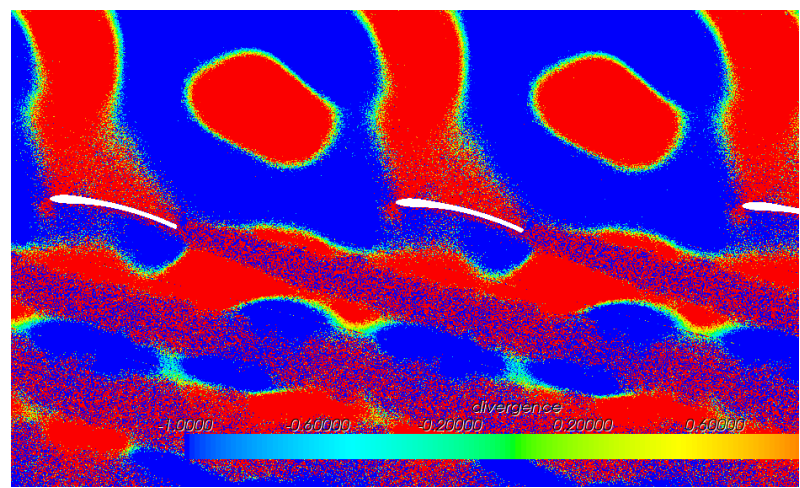

Fig. 12 Divergence of the velocity in a blade cascade. directions for propagation can be observed, depending on the upstream or downstream position. In the first case, the waves are attached to the upper side and propagate in the direction of the blade cascade. In the second case, waves are perpendicular to the first one and move along the initial axis of rotation (i.e. from upstream to downstream).

It must be reminded that the wave length at 5000 $\mathrm{Hz}$ is $64 \mathrm{~mm}$, which is equivalent to the chord length $(60 \mathrm{~mm})$, and thus it is difficult to see if the acoustic wave retro-acts on the vortex shedding at the trailing edge. The TS effect happens in this case while main dimensions of both the geometry and the acoustic waves match. This raises questions about a potential interaction mechanism between the wave and some length of the geometry. No answer could be provided at this stage, but if such effect exists it might explain why some fans are subject to this TS phenomenon while others do not trigger it.

A second simulation for the same profile working at its nominal conditions shows that the vortex shedding still exists (Figure 13, left), but experimental measurements confirm that the acoustic phenomenon disappears, or at least is being so weakened that it is hidden in the classical broadband noise. The sound pressure level (recorded 1.5 chord downstream of the trailing edge) does not show neither a hump (Figure 13 , right). It simply indicates as a first conclusion that the vortex shedding is mainly driven by the profile 


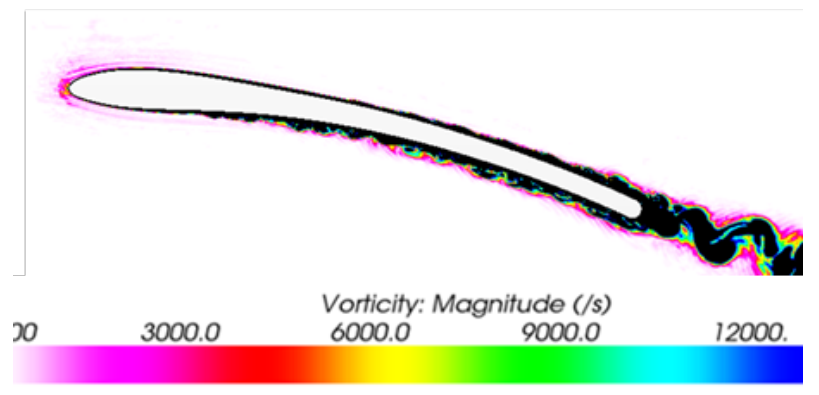

Vorticity at nominal incidence

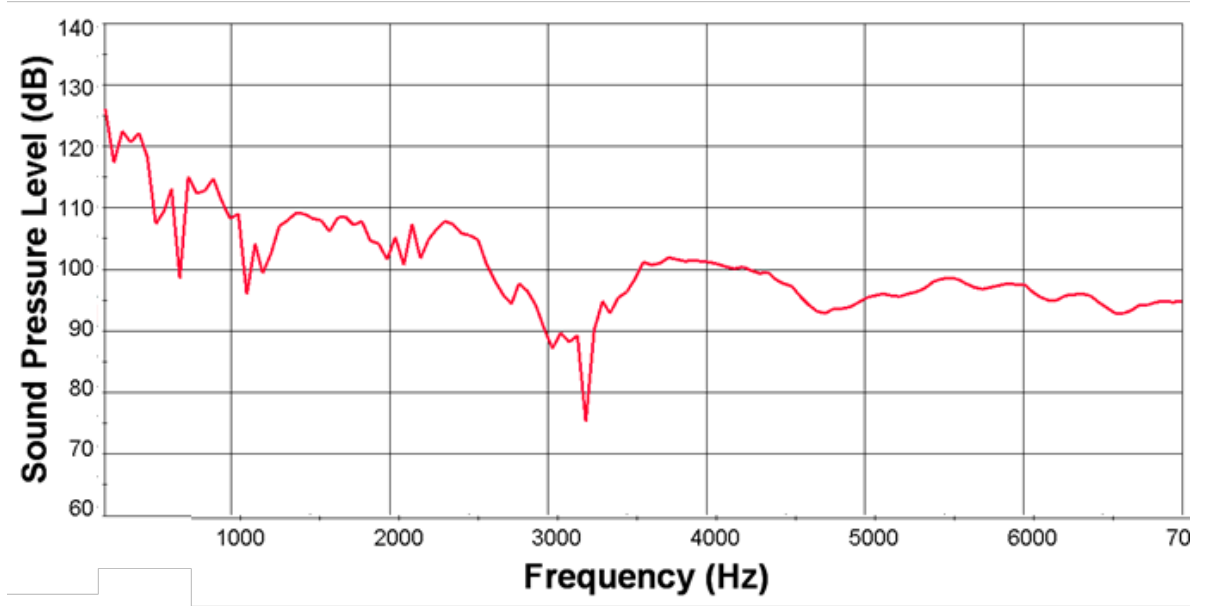

Sound pressure level in the wake 1.5 chord downstream at nominal incidence

Fig. 13 Post-processing at nominal incidence.

thickness, and does not need an acoustic wave to be forced at a fixed frequency.

\section{Geometrical Modifications on the Profile}

As the blade thickness is constrained by the process of production, a modified profile with a different camber has been proposed for investigation. This profile has the same properties than the initial one: identical chord length, inlet and outlet angles and camber height. The only modification concerns the position of its maximum camber height which is positioned closer to the leading edge. The profile comparison on Figure 14 shows that the new one is more loaded at leading edge, with a less smooth curvature on the upper side.

A third compressible LES shows that the flow deviation is almost null at this operating point, and that the vortex shedding is still present in the wake of the profile (Figure 15). The separation on the lower

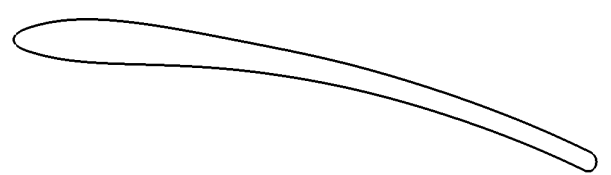

la.

Initial profile

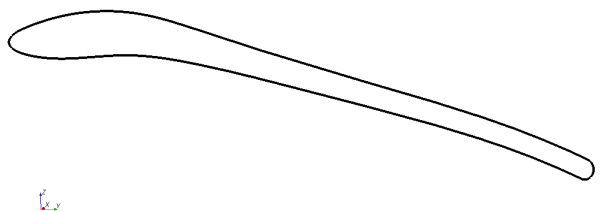

Modified profile

Fig. 14 Initial profile and modified profile.

side is even re-enforced, a larger boundary layer appears on the upper side and the wake still presents vortical structures. As for the initial profile at its nominal operating point, no more hump in the sound pressure level is observed. A link could be assumed between this improvement at high flow rate, close to the zero pressure point, and the forced transition from 


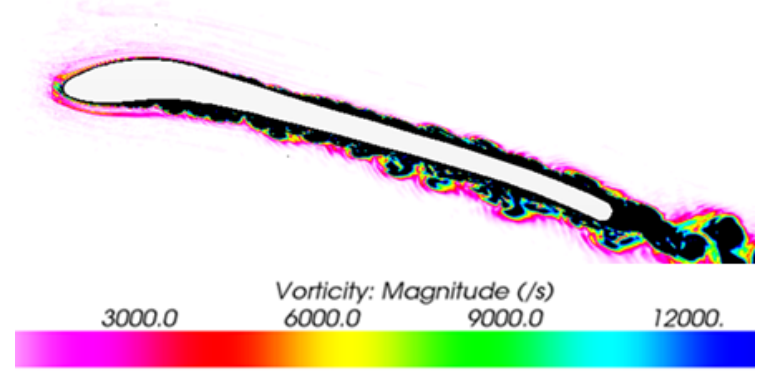

Fig. 15 Vorticity in a plane section on the modified profile.

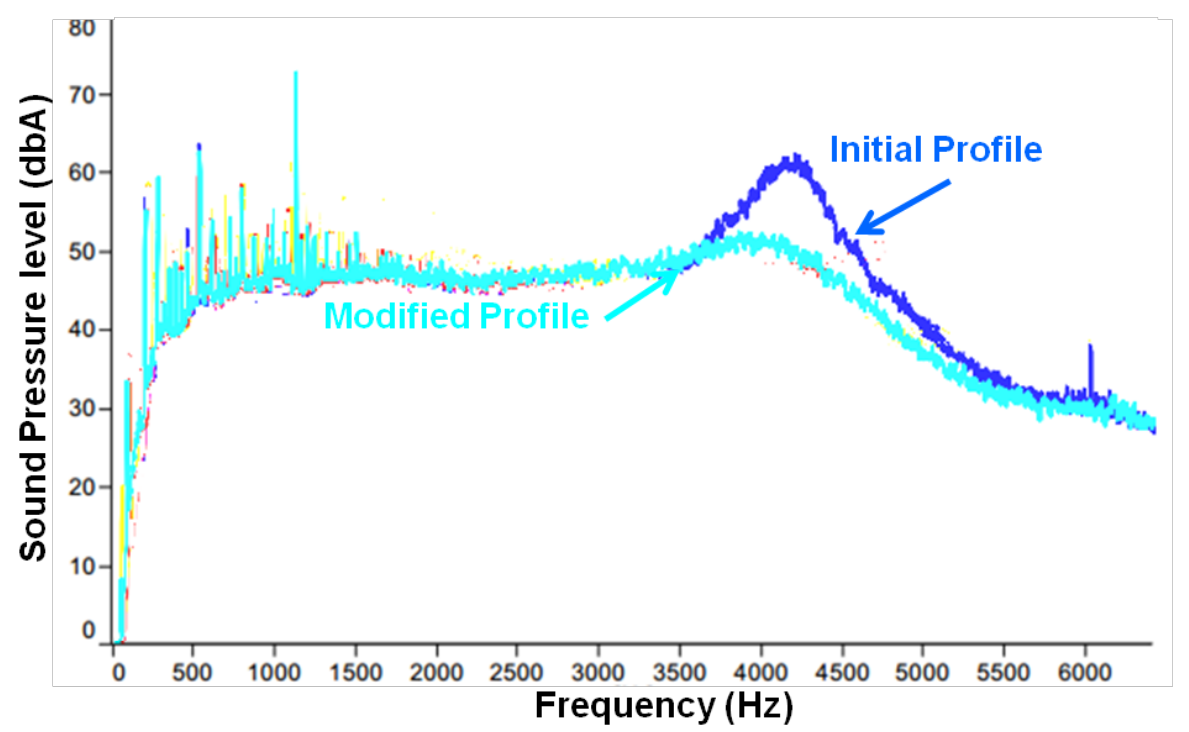

Fig. 16 Compared acoustic spectra for initial and modified profile.

laminar to turbulence just behind the leading edge (by the effect of the camber).

This modified profile has been used to prototype a new fan. Same geometry than the original one is used, meaning same chord length and same stagger angle for bottom to top. Aerodynamic performances are almost equal, the fan efficiency being reduced by only $1.5 \%$. Acoustic measurement presented in Figure 16 shows that the hump shaped resonance is nicely diminished for high flow rate (global level decrease by $3.1 \mathrm{~dB})$.

\section{Conclusions}

An unexpected hump shaped broadband noise has been observed on a fan. Initial investigation (rubber tape) has led to the conclusion that a TS phenomenon occurred on the fan. This phenomenon has been widely described and investigated on profiles, and a previous observation has been reported for an axial fan by Longhouse.

This phenomenon is further investigated thanks to numerical simulation. A compressible LES brings evidence of this acoustic phenomenon, triggered by the vortex shedding at the trailing edge. However, the mutual interaction is not demonstrated, as the same vortex shedding is observed without the acoustic wave either at low flow rate or for a modified profile.

This effect is seldom observed, and the preliminary investigations reported in this paper indicate that the camber is a key factor. Others parameters could be probably found in the $3 \mathrm{D}$ construction of the fan, since this phenomenon is not always observed, or at least not always detected. It might be possible that it is widely encountered at high flow rate, but hidden in the 
global noise and that only few cases lead to a large hump in the frequency domain.

\section{References}

[1] M. Roger, S. Moreau, Broadband self-noise from loaded fan blades, AIAA journal, Vol. 42, No.3, March 2004.

[2] S. Moreau, LES prediction of wall-pressure fluctuations and noise of a low-speed airfoil, International Journal of Aeroacoustics Volume 8, Number 3 / May 2009

[3] F. M. White, Viscous fluid flow, 1991
[4] M.Roger, Coupled oscillations in the aeroacoustics of a Katana blade. Acoustics 08, Paris. 2008

[5] R.E. Longhouse, Vortex shedding noise of low tip speed, axial flow fans. Journal of sound and vibration, 1977

[6] R. W Paterson., P. G. Vogtand,and al.. Vortex noise of isolated airfoils. 1972

[7] M.Henner, B.Demory, F.Franquelin , Test rig effect on performance measurement for low loaded high diameter fan for automotive application. ASME Turbo Expo 2014.

[8] Nicoud, F. and Ducros, F. Subgrid-Scale Stress Modelling Based on the Square of the Velocity Gradient Tensor. Flow, Turbulence and Combustion, 62 